\title{
The Effect of Abdominal Breathing with Muscle Strength Exercise on Balance and Strength
}

\author{
Hyo-lyun Roh \\ Dept of Physical therapy, Kangwon National University
}

\begin{abstract}
Purpose The purpose of this study was to effect of balance ability and the low trunk strength when application the low extremity strength exercise with abdominal respiration exercises. Methods It was conducted for six weeks with 44 healthy persons(male: 25, female: 19) and they were divided 3 groups; control group $(\mathrm{n}=15)$, the lower extremity exercise group $(n=14)$, combined the lower extremity exercise with abdominal respiration $(n=15)$. The exercise program consists of warming up, main exercises and cool down. For each change of motion in the exercise, the experimental group A proceeded to natural breathing group B for 40 seconds. To see the effect of intervention, using Functional Reaching Test for dynamic balance, One Leg Standing for static balance and Primus RS for the low trunk strength. Results Compared before and after the intervention, control group didn't show any differences but group A and group B were improved static balance, dynamic balance and strength of low trunk. There was no differences in the balance and strength between two groups. Conclusion The effect of balance and strength of low trunk were similar to that of the group with abdominal respiration group and did not perform abdominal respiration group. Therefore the combination of abdominal breathing during strength training did not affect muscle strength and balance.
\end{abstract}

Key words Abdominal respiration, Lower extremity, Dynamic balance, Static balance, Strength

Corresponding author Hyo-lyun Roh (bustryagain@nave.com)

Received date 31 January 2018

Revised date 13 February 2018

Accepted date 21 February 2018

\section{Introduction}

The abdominal breathing is a breathing method which consists mainly of contraction of the diaphragm, which is a descending movement of the diaphragm. The abdominal breathing exercise is a breathing technique that slowly sinks deep into the nose and intentionally pushes the abdomen to the belly to allow the air to enter deeply into the belly and ventilates the air through the mouth and nose to ventilate the abdomen intentionally. ${ }^{1)}$

In addition, abdominal breathing has been used to improve the stability of the trunk ${ }^{2)}$ Respiration is the movement of the air in and out of the lungs due to changes in the volume of the thorax. Especially, the muscles of the abdominal muscles work together as diaphragm partners. The contractions of the muscles act on strong exhalation ${ }^{3)}$ In this study, the effect of

doi : http:dx.doi.org/10.17817/2018.02.04.111238 muscle activity on muscle activity was examined.

The diaphragm and stomach contributes to the postural control with breathing and the activity of the muscles in the diaphragm and stomach occurs before other limb movements occur. In addition, the diaphragm, when the balance of the trunk is unstable, contracts and contracts together with the muscles adjacent to the abdominal cavity and the abdominal cavity to harmonize the movement of breathing, abdominal muscles and thorax, and contributes to stabilization of the vertebrae.

Stability and balance are necessary to maintain diverse attitudes in everyday life. In addition, abdominal muscles and lower body muscles are related to trunk stability and are important for movement and posture control. The loss of selective movement of the trunk muscles implies a decrease in stability to maintain an upright posture, which is closely related to muscles and respiratory muscles to maintain posture. ${ }^{4)}$ Judge et 
al $(1993)^{5)}$ reported that muscle weakness affects balance and muscle strength is needed to overcome postural instability.

There have been many studies to improve posture instability and balance, but there is lack of research on abdominal breathing, balance, and lower limb muscle strength. The purpose of this study is to investigate the effect of abdominal breathing on balance ability and lower limb muscle strength.

\section{Materials and Methods}

\section{Subjects and Research procedure}

The subjects were 20 male and 19 female healthy adults. They were randomly divided into three groups according to the selection criteria. The control group consisted of 9 males and 6 females in total 15 $(24.20 \pm 1.57$ year, $70.47 \pm 19.0 \mathrm{~kg}, 171.43 \pm 8.48 \mathrm{~cm})$. In the experimental group A, a total of 14 subjects $(21.57 \pm 1.90$ year, $69.65 \pm 16.64 \mathrm{~kg}, \quad 167.13 \pm 6.25 \mathrm{~cm})$ were used to perform the exercise program on the mat without abdominal breathing. Experimental group B consisted of 8 male and 7 female patients in total $15(21.87 \pm 1.55$ year, $60.71 \pm 9.59 \mathrm{~kg}, 167.77 \pm 5.72 \mathrm{~cm})$ with combined abdominal breathing and exercise program on the mat. Two groups performed the exercise program for total of 6 weeks.

The criteria for selection of the subjects were those who have not undergone lower limb surgery, those who have not had an injured leg or ankle sprain for the past year, were not injured in the trunk by traffic accidents. All subjects were informed about this study, received the volunteer consent, participated in the experiment, and informed that the subject can quit midway if they did not want to participate in the study. Participants in the study conducted a preliminary exercise to perform only the activities during daily life without additional exercise or endurance exercise other than the exercise program presented in this study.

The exercise program consists of 5 minutes of warming up, 30minutes of main exercises and $5 \mathrm{mi}-$ nutes of cool down. The preparation exercise was performed for 5 minutes in total for 5 minutes for each 1minute. The exercises were performed with squats, side leg raises, leg bridges, lunge, calf raises, dead lifts and side lunge sets. Each time the operation was changed, the experimental group A performed natural breath breathing group B for 40seconds. The finishing exercise was performed for 5 minutes in total for 5 minutes. The abdominal breathing posture was performed so that one hand was located in the dress bone part and the other hand was located in the navel area to recognize the correct abdominal breathing.

\section{Measurement tools}

Static and dynamic balance were tested in the obese volunteers with one leg standing and functional reach tests. Static standing balance was tested with one leg standing under the eyes open). Standing still for longer periods of time represents better static standing balance. The functional reach test was used to investigate dynamic standing balance by determining the farthest distance subjects could possibly reach forward.

Lower limb muscle strength was measured using Baltimore Therapeutic Equipment (BTE, P41AL30A, Hanover). BTE Primus RS can to measure isokinetic and isometric strength. In order to measure the lower limb muscle strength, the subject was sitting on a chair without a backrest, crossed with both hands, placed on both shoulders, and fixed with a belt. The examiner asked the subject to use only the lower trunk muscle strength. To obtain the resistance value, 3 isometric measurements were made and each measurement had 6 seconds of rest time. The muscle strength was measured 2 times for each 10 seconds, and the resting time was 10 seconds. The average muscle strength of the lower body and bending of the lower body was recorded.

\section{Data analysis}

SPSS 21.0 for Windows (IBM Corp., Armonk, NY, USA) was used for data analysis. To examine the balance and strength, paired t-tests were conducted for comparison within groups. A one-way ANOVA was used for among groups comparisons, while the Tukey test was used for post-hoc analysis. The Statistical significance was set at $\mathrm{p}<0.05$. 


\section{Results}

\section{Measurement dynamic balance ability}

We examined the degree of changes of functional reach test before and after intervention in control group, experimental group A (lower exercise group), and experimental group B (double breathing + lower exercise group). In the control group, functional reach test arm was reduced after intervention rather than before intervention. There was a statistically significant difference between the experimental group A and the experimental group B $(\mathrm{p}<0.01)$ (Table 1$)$. Therefore, the experimental group $\mathrm{A}$ and the experimental group $\mathrm{B}$ seem to have improved the dynamic balance after intervention.

\section{2) Measurement static balance ability}

We examined the degree of change of the control group, the experimental group $\mathrm{A}$, and the experimental group $\mathrm{B}$ before and after the intervention. There was no statistically significant difference between the control group and the experimental group
A $(\mathrm{p}<0.05)$ and the experimental group B $(\mathrm{p}<0.01)$ (Table 2). Therefore, the experimental group $\mathrm{A}$ and the experimental group B seem to have improved the static balance after the intervention.

\section{3) Measurement lower strength}

3 groups were examined to lower strength by BTE before and after the intervention. There was no statistically significant difference in the control group, but there was a statistically significant difference between the experimental group $\mathrm{A}(\mathrm{p}<0.05)$ and the experimental group $\mathrm{B}(\mathrm{p}<0.01)$ (Table 3$)$. Therefore, it seems that the muscle strength of the lower body after the intervention in the experimental group $\mathrm{A}$ and the experimental group B is improved.

\section{4) Comparison between groups before and after intervention}

The functional arm stretch between the three groups, one leg leg clenching test, and the difference in the degree of change in lower limb muscle strength were examined.

Table 1. Changes in functional reaching test

\begin{tabular}{|c|c|c|c|}
\hline \multirow{2}{*}{ Group } & \multicolumn{2}{|c|}{ Mean $\pm S D$} & \multirow{2}{*}{$\mathrm{t}$} \\
\hline & pre & post & \\
\hline Control group & $20.94 \pm 5.22$ & $19.75 \pm 4.65$ & $3.127^{* * *}$ \\
\hline A group & $16.82 \pm 5.11$ & $23.00 \pm 7.14$ & $-4.088^{* * *}$ \\
\hline $\mathrm{B}$ group & $15.66 \pm 5.27$ & $26.93 \pm 3.98$ & $-8.572^{* *}$ \\
\hline
\end{tabular}

** $\mathrm{p}<0.01$, A group : Lower extremity exercises group, B group : Lower extremity exercises and Breathing exercises group

Table 2. Changes in one leg standing

\begin{tabular}{|c|c|c|c|c|c|}
\hline \multirow{2}{*}{ Group } & \multirow{2}{*}{ Side } & \multicolumn{2}{|c|}{ Mean \pm SD } & \multirow[b]{2}{*}{$\mathrm{t}$} & \multirow[b]{2}{*}{$\mathrm{p}$} \\
\hline & & pre & posr & & \\
\hline \multirow{2}{*}{ Control group } & Rt. & $15.76 \pm 8.88$ & $16.83 \pm 9.11$ & -2.117 & 0.053 \\
\hline & Lt. & $15.31 \pm 8.79$ & $15.44 \pm 7.74$ & -0.195 & 0.848 \\
\hline \multirow{2}{*}{ A group } & Rt. & $6.14 \pm 4.13$ & $28.40 \pm 26.67$ & -3.421 & 0.005 \\
\hline & Lt. & $9.47 \pm 7.24$ & $28.47 \pm 28.45$ & -2.816 & 0.015 \\
\hline \multirow{2}{*}{ B group } & Rt. & $8.17 \pm 5.82$ & $20.71 \pm 18.85$ & -3.290 & 0.005 \\
\hline & Lt. & $7.67 \pm 6.21$ & $25.40 \pm 16.44$ & -5.509 & 0.000 \\
\hline
\end{tabular}

A group : Lower extremity exercise group, B group : Lower extremity exercise and Breathing exercise group 
Table 3. Changes in baltimore therapeutic equipment

\begin{tabular}{|c|c|c|c|c|c|}
\hline \multirow{2}{*}{ Group } & & \multicolumn{2}{|c|}{ Mean士SD } & \multirow[b]{2}{*}{$\mathrm{t}$} & \multirow[b]{2}{*}{$p$} \\
\hline & & pre & post & & \\
\hline \multirow{2}{*}{ Control group } & flex. & $29.62 \pm 12.18$ & $29.6 \pm 11.62$ & 0.039 & 0.970 \\
\hline & ext. & $25.76 \pm 9.78$ & $25.39 \pm 9.51$ & 0.654 & 0.523 \\
\hline \multirow{2}{*}{ A group } & flex. & $12.6 \pm 8.18$ & $17.74 \pm 8.56$ & -2.556 & 0.024 \\
\hline & ext. & $16.42 \pm 8.78$ & $23.72 \pm 11.14$ & -2.953 & 0.011 \\
\hline \multirow{2}{*}{ B group } & flex. & $9.73 \pm 7.42$ & $18.09 \pm 11.63$ & -4.069 & 0.001 \\
\hline & ext. & $18.84 \pm 14.61$ & $35.14 \pm 22.12$ & -5.156 & 0.000 \\
\hline
\end{tabular}

${ }^{*} \mathrm{p}<0.05,{ }^{* *} \mathrm{p}<0.01$, A group : Lower extremity exercise group, B group : Lower extremity exercise and Breathing exercise group

Table 4. Compared intra group

\begin{tabular}{|c|c|c|c|c|c|c|}
\hline & & Control group ${ }^{a}$ & A group ${ }^{b}$ & B group ${ }^{c}$ & $\mathrm{~F}$ & post test \\
\hline FRT & & $1.19 \pm 1.48$ & $-6.18 \pm 5.66$ & $-11.27 \pm 5.09$ & $29.810^{\text {***** }}$ & $a>b>c$ \\
\hline \multirow{2}{*}{$\begin{array}{l}\text { One leg } \\
\text { standing }\end{array}$} & Rt. & $-1.07 \pm 1.96$ & $-22.25 \pm 24.34$ & $-12.54 \pm 14.76$ & $6.194^{* * * * *}$ & $\mathrm{ac}>\mathrm{bc}$ \\
\hline & Lt. & $-0.13 \pm 2.67$ & $-19.00 \pm 25.25$ & $-17.73 \pm 12.46$ & $6.386^{* * * *}$ & $a>b c$ \\
\hline \multirow{2}{*}{ BTE } & Flex. & $0.02 \pm 1.99$ & $-5.14 \pm 7.53$ & $-8.37 \pm 7.96$ & $6.544^{* * * * *}$ & $a>b c$ \\
\hline & Ext. & $0.37 \pm 2.17$ & $-7.30 \pm 9.25$ & $-16.30 \pm 12.24$ & $13.059^{* * * * *}$ & $a>b>c$ \\
\hline
\end{tabular}

*** $\mathrm{p}<0.001$, A group : Lower extremity exercise group, B group : Lower extremity exercise and Breathing exercise group

In the functional arm stretch, there was a statistically significant difference between the three groups ( $p$ <0.01). In addition, there was a statistically significant difference between the groups ( $p<0.01)$, and there was a statistically significant difference between the groups in the degree of postural change of the lower limb muscle strength between the groups $(\mathrm{p}<0.01)$. Therefore, the dynamic balance and static balance between the three groups and the degree of change in the lower body seem to be different.

\section{Discussion}

The purpose of this study was to investigate the effect of abdominal breathing on balance ability and lower limb muscle strength.

In this study, the degree of dynamic and static balance was improved by increasing the degree of func- tional stretch test and limb clenching after 6 weeks of exercise in the lower limb group, the lower extremity group, and the abdominal breath group. In the study of Lee $(2001)^{6}$, the results of this study were supported by reporting a high correlation between balance and leg strength. In addition, the exercising that strengthens leg strength is important for improving balance. Nasher(1990) ${ }^{7)}$ reported that balance ability was improved with better muscle strength in research on the relationship between strength and balance ability. We have studied the effects of exercise and reported improved balance ability after exercise than before exercise(Brill et al, 1998) ${ }^{8}$. Judge et al(1993) ${ }^{5)}$ reported that the greater the muscle strength, the better the ability of one leg clerk. In the study of Balancing ability is directly related to lower limb strength, and weakening of lower limb strength affects the whole daily life including stepping, stair climbing, and standing in the chair ${ }^{9}$. Therefore, this study suggests that both the costume and the strengthening exercises of 
the lower limb are useful for improving balance and leg strength.

In this study, lower limb muscle strength was increased in lower limb group and abdominal breathing with lower limb exercises group. According to $\operatorname{Kim}(2006)^{10)}$, the results of this study were supported by the fact that abdominal breathing is related to activation of lumbar muscle. According to Kwak et al. $(2010)^{11)}$, abdominal respiration is related to abdominal obesity rate and abdominal breathing alone contributes to activation of abdominal muscles. The lower limb muscles are the centers where all the forces and movements of the human body are made, centering the limbs and keeping the musculoskeletal structure appropriately ${ }^{12}$. The muscles of the lower trunk are those that align and maintain their postures while participating in the movement of the proximal part, thereby enhancing the effect of the distal part ${ }^{13)}$, which plays a central role in functional activities and is also the basis or motive force of all limb movements in terms of medicine. This provides stability through the simultaneous contraction of the muscles around the trunk, such as the role of the support rope to support the stability of the tent. Stability enhancement of the lower triceps effectively induces movement of the limbs during exercise ${ }^{14)}$. If the lower limb stability is secured, the activities of the abdominal muscles, the waist, the pelvis and the hip joints will be harmonized and the function of the limbs will be made more smoothly and the supporting function of the spine will be provided.

In this study, differences were also found between lower extremity exercise group and lower extremity with breathing group. In the one leg test, which measured the static balance, the degree of change in the lower limb movement group was greater, and in the functional reach test, which measured the dynamic balance, the change in the group with the lower extremity exercise and the abdominal breathing group was greater. Respiratory imbalances can potentially lead to changes in body balance ${ }^{10)}$, and muscle-strengthening exercises combined with double breathing seem to be effective in maintaining dynamic balance. In addition, the degree of muscle strength of the lower trunk showed a greater degree of change in both the bending and swelling groups, which were accompanied by lower limb movement and abdominal breathing. Therefore, it seems that abdominal breathing contributes to strengthening muscular strength. In a study by Sutbeyaz et al.(2010) ${ }^{1)}$, it was reported that abdominal breathing improves exercise performance and respiratory function, increases muscle strength of respiratory muscles, and maintains and improves chest and vertebral mobility were similar.

In the area of physiotherapy, abdominal breathing can be used in various age groups, but abdominal respiration is not a big part in physical therapy. Therefore, in future studies, when exercise and abdominal breathing are performed in parallel, researches that can support abdominal breathing effect should be carried out by adding abdominal breathing group.

\section{Conclusion}

The purpose of this study was to investigate the effects of abdominal breathing and lower limb exercise on balance and lower limb muscle strength in healthy adults.

Balance and strength were improved in the lower limb exercise group only and abdominal breathing with lower limb exercise group. There was no difference between the two groups. Therefore, it seems that abdominal breathing does not contribute to the improvement of balance and lower limb muscle strength.

\section{References}

1. Sutbeyaz ST, Koseoglu F, Inan L, et al. Respiratory muscle training improves cardiopulmonary function and exercise tolerance in subjects with subacute stroke: a randomized controlled trial. Clin Rehabil. 2010;240-50.

2. Kolar P, Sulc J, Kyncl M, et al. Stabilizing function of the diaphragm: dynamic MRI and synchronized spirometric assessment. J Appl Physiol. 2010;109 (4):1064-71.

3. Kaneko H, Satou H, Maruyama H. Reliability of lateral abdominal muscles thickenss measurement using 
ultrasonography. Rigakuryoho Kagaku. 2005;20(3): 197-201.

4. Wang YT, Kent RD, Duffy, et al. Dysarthria in traumatic injury: A breath group and intonational analysis, Folia Phoniatr Logop. 2005;57(2):59-89.

5. Judge JO. Balance improvements in older women effects exercise training. Phys ther. 1993;73(4):254-65.

6. Lee DH, Ro HL. The effects of increase in balance ability to one leg support exercises for adults using empty bottle. J Kore Socie Neurotheapy. 20143;17(1):1-5.

7. Nasher LM. Sensory, neuromuscular, and biomechanical contributions to human balance. APTA Publications. 1990;1-12.

8. Brill PA, Probst JC, Greenhouse DL, et al. Clinical feasibility of a free-weight strength-training program for older adults. J Am Board Fam Pract. 1998;11(6):445-51.

9. Manini TM, Visser M, Park W, et al. Knee extension strength cutpoints for maintaining mobility. J Am Geriatr Soc. 2007;55(3):451-7.

10. Kim K. Effect of diaphragmatic breathing exercise on activation of lumbar paraspinal muscles of healthy people. 2006;1(1):59-66.

11. Kwak YS, Kim YI. The effect of abdominal breathing exercise on weight and body fat, BMI, waist hip ratio in obese college student. J Life Scie. 2010;20(12): 1867-187.

12. Kisner C, Colby LA. Therapeutic Exercise: Foundations and techniques. 4th ed. Philadelphia, PA, F.A. Davis Co., 2002:63-5.

13. Akuthota V, Nadler SF. Core Strengthening. Arch Phys Med Rehabil. 2004;85(1):86-92.

14. Gambetta V, Clark M. Hard core training. Training and conditiong. 1999;9(4):34-40. 